\title{
Interactive Water Resources Modeling and Model Use: An Overview
}

\author{
DANIEL P. LOUCKS \\ School of Civil and Environmental Engineering, Cornell University, Ithaca, New York
}

JANUSZ KINDLER

Institute of Environmental Engineering, Warsaw Technical University, Poland

\author{
KURT FEDRA
}

International Institute for Applied Systems Analysis, Laxenburg, Austria

\begin{abstract}
This serves as an introduction for the following sequence of five papers on interactive water resources and environmental management, policy modeling, and model use. We review some important shortcomings of many management and policy models and argue for improved human-computer-model interaction and communication. This interaction can lead to more effective model use which in turn should facilitate the exploration, analysis, and synthesis of alternative designs, plans, and policies by those directly involved in the planning, management, or policy making process. Potential advantages of interactive modeling and model use, as well as some problems and research needs, are discussed.
\end{abstract}

\section{INTRODUCTION}

We recently conducted an informal survey. We asked many of our acquaintances in government agencies concerned with water resources and environmental protection what their biggest problems seemed to be. All of their responses could be summarized as keeping ahead of the increasing number of water- and environmental-related "crises." Efforts spent in reducing each current crisis seems only to result in the emergence of seemingly more difficult ones.

In spite of all that has been done to reduce the ever reoccurring cycle of floods and droughts and their accompanying damages, these events still occur, and their damages are increasing. On top of these and other reocurring problems it seems, suddenly, we have a host of new problems. Increasingly, various toxic wastes discharged on or under the ground are contaminating groundwater supplies and occasionally oozing up to the surface to create health hazards for those living on it. Our urban water distribution systems, many that have functioned well beyond their design life, have collectively, it seems, decided to call for help. The control of nonpoint source pollution is becoming a major challenge to surface water quality managers. Increasingly, acid rain, in a variety of wet and dry forms, is adversely altering aquatic and forest ecosystems on regional scales. The potential for more Three Mile Island nuclear accidents is becoming increasingly apparent, and so on. Any reading of major daily newspapers could lead one to conclude that we are not yet very effective managers of our water and other natural environmental resources. We ought to be able to do better, and we can if we have the will.

What is to be discussed in this and the following sequence of papers is not going to solve all of our current water and environmental problems. However, we believe it can provide some help toward that end. It seems to us that managers, planners, and policy makers could benefit from having avail-

Copyright 1985 by the American Geophysical Union.

Paper number 4 W0977.

0043-1397/85/004W-0977\$05.00 able, when they need it, some easy-to-use tools to help them identify, explore, analyze, and synthesize effective resource management plans and policies.

The development and application of management or policyoriented models for helping water and environmental resource managers have been taking place for several decades throughout much of the world. The extent to which this effort has been as useful as it could have been is in some doubt. A comprehensive study of the use of models for water resource management, planning, and policy in the United States was recently completed by the Office of Technology Assessment (OTA) of the U.S. Congress [Friedman et al., 1982]. This report emphasizes the potential of currently existing models for improving the accuracy and effectiveness of informaton available to managers, decision makers, and scientists. It also documents the need for models in meeting the requirements of a wide variety of Federal and State laws in the United States concerning resource management and environmental protection. However, the primary focus of the report is on the constraints to effective model use. Among these constraints are the lack of information about available models, lack of training in model use and interpretation, lack of communication between model users and developers, and lack of required support services.

- Some constraints to model use can only be reduced by actions taken by potential user agencies or groups. Much of the OTA report is directed toward this issue and suggests possible courses of action that resource management agencies could take to increase the benefits they can obtain from model use. However, there are other constraints that we as modelers and analysts can help to reduce. It is this need and opportunity that motivates the discussion in this and the following papers.

Many of the constraints for effective model use that confront us today are the same that Little [1970] identified well over a decade ago. Not every proposed model is appropriate in all contexts. A very good model for one problem and institutional setting may not be as good, or even applicable, in another. Unless model builders are familiar with both the problem and the institutional setting in which the problem is 
to be addressed, it is unlikely any modeling work will be very effective. Good model parameterization and validation is rare. Models must be made credible to the potential users. Regardless of whether or not the users fully understand the model or models, the outcome must be believable. It must conform to the user's perception of the world or at least that part of it being modeled. Finally, the model must be complete. It must include the capability of examining all of the issues deemed important by the user, and the resulting information must be easy to understand. How to make our models more flexible or adaptable to alternative paths of exploration and how to design an interface for more effective communication between the user and the model are continuing challenges to model builders.

Our thesis, in short, is as follows. Modeling, if it attempts to describe more than some microaspects of physical systems, by necessity involves a strong subjective and value-dominated human element which defies a formal representation in any generally acceptable way. At the same time, any acceptable formal model must be compatible with the set of mental models and the cognitive styles of their users. We argue that widespread model acceptance and use can only stem from direct user involvement at various phases of the modeling process, including definition of basic assumptions and the control of output formats. Interactive methods which give the user an appropriate role in controlling model calibration, model use, and output display requires new ways of manmachine interaction. The role of models thus changes from a product-oriented function where models are products of a usually external modeling process and produce solutions which the end-user does or does not accept, to a process-oriented function. In a process-oriented function, modeling and model use are the important elements of a learning process which in turn facilitates the policy and decision-making process.

This paper, and those that follow, present some ideas and general approaches for model builders to consider as they work toward improving the effectiveness of their models to managers, planners, and policy makers. Given the increasing number and complexity of today's water and environmental management problems and those that are likely to emerge in the future, it follows that the potential utility of models designed to help study these problems is also increasing. Clearly, the demand for information that can and should be derived from models exists; it is only a question of how well we as model builders can meet it [Brown, 1982]. We will need, and we will develop, better models, of course, as our knowledge of the systems we model increases. However, equally important, we must devote some attention to the interface between the model user and the models being used. More effective communication is essential for increased effectiveness in model use.

Many gaps between model availability and model use can be reduced by the development of a more user-friendly interaction between the model and its user. Facilitating humanmodel-computer interaction will not necessarily increase the likelihood that any one or more model solutions will be implemented. Rather, it should, we believe, increase the likelihood of the models producing information useful in any debate over what to do, who will be affected, how they will be affected, and how much they will care.

Models, if and when used in the planning or policy making process, are used because they provide and organize information that together help in the understanding of the problems being addressed. Often this "additional" information may simply better define the problem and the possible conse- quences of particular actions taken to help solve or reduce it [Little, 1970]. It may also assist in the resolution of conflicts by providing negotiators with a "face-saving" excuse for changing their positions, i.e., for compromise [Sebenius, 1981; Straus and Clark, 1980; Lara and Sachs, 1978].

Before discussing our proposed interactive approach to modeling and model use it seems appropriate to first briefly discuss in a general way how much of water resources and environmental policy making seems to take place. This discussion provides a context for comparing what we are proposing with some past approaches to policy modeling that placed little if any emphasis on human-model-computer interaction and communication.

\section{Some Characteristics of Planning and Policy Making}

Water resources and environmental planning and policy making are commonly characterized by their breadth of impact, uncertainty, scarcity of causal evidence on which to base a plan or policy, and by their multiobjective and multiinstitutional involvement. Many individuals, interest groups, and organizations are affected and concerned about water and the environment. We are not very good at predicting future economic conditions, costs, and benefits, or estimating future social or political impacts of current decisions, plans, or policies. We must use considerable subjective judgment to supplement what meager evidence might exist to help us predict even the physical, biological, or chemical impacts of alternative decisions. Finally, water and environmental problems tend to be heavily value-laden, and objectives and institutions are very much a part of the problem.

As a consequence of all this apparent confusion and uncertainty, water resources and environmental problem-solving tends to be an iterative, explorative, learning process that redefines the problems as much as it seeks solutions to them. Any review of the U.S. water pollution control policies over the past several decades, for example, will provide ample evidence of this.

The very nature of this iterative, recursive, learning, and decision-making process usually requires subjective evaluations and considerable compromises among conflicting goals. The process is typically more political than analytical or scientific. The direct involvement of various interest groups is common. Their goals and behavior are not easily modeled in any acceptable way. What model builders may assume as rational cannot be assumed. What is rational to one may not be to another, and for reasons that may not ever be revealed.

Values and beliefs, and the resulting objectives that shape policies, are the result of dynamic human and institutional interactions. While one could suggest, therefore, that "planners must design from the beginning for the complete range of objectives" [Kahn, 1960], such a task may be difficult to accomplish. Objectives and values are usually conflicting and changing. They are dependent on the choices given, i.e., on the status of the learning process. Values may very well be unknown to those who have them; i.e., one may not be able to make them explicit when asked. Yet the same person will usually be able to select preferred plans or policies from among alternatives.

Values and priorities may also depend on the possibilities and tradeoffs in a bargaining situation. Such situations are typical in multiple objective decision making exercises that characterize water resources and environmental planning and policy making. Goals may change and unknown constraints may emerge during such processes. 
To include these complex and uncertain behavioral characteristics of planning and policy making within one or more models becomes impossible, but to exclude them from consideration during the development of policy models invites a high chance of model failure or rejection. So, what to do? First, we suggest, is to include within models only those parts of the problem under study which can be modeled with credibility. That which cannot, i.e., most human or institutional behavior, must be left outside the model. This then requires that the models be capable of directly and interactively involving humans, i.e., the managers, planners or policy makers, or their staffs, who must supply the crucial behavioral elements. This capability requires, in turn, that the model or models must fit into the managing, planning, and policy-making processes. They must be appropriate for the institutions in which such processes take place and for the individuals within those institutions involved in managing, planning, or policy making.

Inadequate data of the kinds most needed is another troublesome characteristic of most water and environmental planning or policy-making exercises. Each discipline involved in such multidisciplinary exercises tends to collect data in its own traditional way. Data compatibility can be a major constraint. Temporal and spatial resolution, and the resulting statistical quality as well as the appropriateness of the data differ widely when considering, for example, ecological, geographical, or economic and fiscal data related to a given problem.

Some data in some cases may be available in large quantities. Meteorological and hydrological records and data resulting for remote sensors may be available with considerable temporal and spatial coverage. In such situations, data-related problems are often those of data reduction, aggregation, and interpretation. Data on causal relationships, however, are usually scarce. This is particularly so when the phenomena of interest are the long-term and synergistic impacts of a particular plan or policy pertaining to, for example, public health, economic development, and ecological systems. Once again, decisions in the absence of adequate evidence of causal relationships will be largely based on subjective judgments.

How can these and other troublesome features of water resources and environmental management and policy making be reduced by those who develop models? We believe there needs to be a change in the manner we approach such problems. Before discussing these proposed changes a brief review of our view of how most models have been developed in the past may serve as a basis for comparison.

\section{Traditional Approaches to Policy Modeling}

Many modelers have, perhaps until relatively recently, been following a modeling approach that has focused mainly on the analysis of alternatives and less so on the generation, exploration, and synthesis of alternatives. Many of these models have been designed for use outside of the planning, management, and policy-making process. They were designed for use by policy model builders and analysts to provide quantitative solutions to specific problems. This information has occasionally ended up in one or more reports. Very often these quantitative solutions have been based on the assumption of rational economic behavior for lack of any better assumption. Multiobjective models have been used to attempt to eliminate from further consideration solutions that were not efficient i.e., that were inferior with respect to the objectives considered in the models. The trouble is that rational behavior of any kind is not likely to be the same among different individuals and that unspecified, perhaps unknown and unquantifiable, objectives and constraints also apply.

The difficulties in identifying appropriate policy objectives is increasingly being recognized. Many models found in the literature assume the existence of one or more fixed objectives. In the case of prespecified multiple objectives, one can resort to multiobjective or multicriteria modeling to generate sets of "efficient," "noninferior," or "Pareto-optimal" solutions [Cohon, 1978; Zeleny, 1981a, b; Goicoechea et al., 1982]. However, solving large multiobjective models many times to generate even a small subset of noninferior solutions has proven to be an expensive and time-consuming effort. Moreover, there is increasing realization that individuals may want to consider an apparently inferior solution (with respect to those objectives being modeled) as well as noninferior ones. So-called inferior solutions may not be inferior at all when considering additional objectives. Rarely, if ever, can all relevant objectives be explicitly considered in multiobjective models. Hence there are legitimate needs to focus on more than only the set of noninferior or efficient solutions generated by multiobjective models.

Perhaps one of the biggest reasons for model solution rejection, even as a basis for discussion in the managing, planning, or policy-making process, has been the lack of model understanding or confidence, and the lack of adequate communication between the analysts and their clients. Throughout the managing, planning, or policy-making process, analysts rarely can address the right questions that managers, planners, and policy makers want answered unless they are aware of these questions. Also, individuals often do not know what questions they want answers for before some exploration and comprehension of the impacts of some of their ideas for plans and policies. The outcome of this exploration will lead to new questions and the need for additional exploration.

Another tendency in the past, and to some extent even today, has been to concentrate on the development of fairly complex and comprehensive models. This is natural. Most water and environmental problems and their impacts are complex and involve a wide variety of individuals, interest groups, and institutions. The trend toward bigger and more comprehensive models which simultaneously try to describe as many relevant aspects of the problem as can be put into the computer has coincided with the growth in computer speed and capacity, and in the development of more efficient algorithms for solving a variety of mathematical models. Large complex models, however, are not only more difficult to develop and maintain; they tend to require more input data, and this can be expensive to obtain. They also tend to produce large quantities of output, much of which can be a challenge to comprehend.

Figure 1 is an admittedly very simplified illustration of many past and even some current attempts of comprehensive model building and application. The heart of the modeling process revolves around the analysts (model builder and user) and the comprehensive simulation and/or optimization model. The potential client, the potential user of model information, is only weakly involved, usually through reports the analysts may prepare based on information derived from the model.

Typical of many resource management and policy-modeling applications, the analysts have some initial contact with those needing information to be obtained from models, e.g., managers, planners, and policy makers or their staffs. They also have some contact with them at the very end when some reasonable results from the models are available. During most 


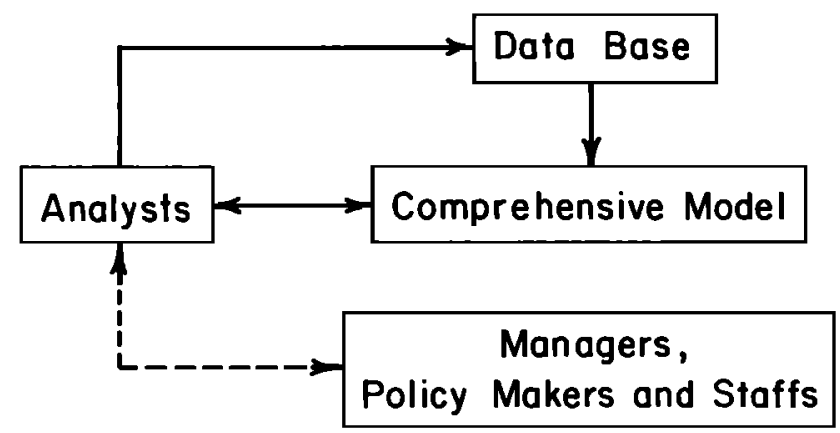

Fig. 1. Typical relationships among managers and policy makers, analysts, their model, and their data, indicating weak or nonexisting links between potential users of model information and the models and those who develop them.

of the intervening time, however, the analysts and potential users tend to go their separate ways. Once some model results are available, the original resource use conflicts, management questions, policy objectives, and sometimes even the managers, planners, or policy makers are often quite different than when the modeling work began. All too often, too little attention has been devoted to building the models into the dynamic management, planning, or policy-making process [Walker, 1982; Lara and Sachs, 1978]. This has made it difficult for anyone but analysts to use models to explore their own ideas, obtain information about the impacts of new policy options, and indeed, to synthesize as well as to analyze alternatives.

The fact that many of our past models were complex and relatively comprehensive did not necessarily have to mean that they were also less comprehendable or less credible to a potential user. That this has tended to be the rule rather than the exception indicates only that in many cases not enough attention has been placed on model management and model use. The challenge for the future is to learn how to model complex systems so that the models, or more likely a system of more comprehendable models, and the interface between the models and the users becomes more intelligible, manageable, useful, and reliable [Quade and Miser, 1983]. Too little attention has been devoted to building models into the dynamic management, planning, and policy-making processes, i.e., model and implentation and communication.

\section{AN APPROACH to MODEl IMPLEMENTATION AND COMMUNICATION}

Any approach to overcoming many of the difficulties and limitations of many of our past models just discussed must recognize the need to involve representatives from each appropriate level of decision making in the modeling process. Involvement of planners and the staffs of policy makers, rather than analysts trying to guess and model their objectives, values, and behavior, is the only way we see how models are going to become more effective as tools for exploring and understanding key issues and for identifying possible management or policy options and their probable impacts. This involvement of nonmodelers in model use, if not also model building, will only happen if the interface between the computer, the models, and the model users makes models easy to use and their results easy to obtain and understand. This interface must permit easy interaction between the model user and the model or models being used. It must make data input and editing easy to perform and make data output easy to manage and comprehend.

Increasing availability of interactive computer hardware and software makes this approach much more feasible today than it was even several years ago. The current revolution in the development of mini- and microcomputers, interactive software, and color graphics is making it possible for each of us, as individuals or as members of an organization, to have access to fast, cheap, interactive (flexible) computing power, supplemented increasingly by graphical or pictorial display capabilities if desired [Weger, 1981; Whitted, 1982]. We are convinced this technological development cannot but have a profound effect on how we all approach policy model building and use in the future. Other articles [Fedra and Loucks, this issue; Loucks et al., this issue] discuss this subject in more detail.

For management, planning, and policy studies, analysts are increasingly beginning to develop systems of interrelated smaller models rather than single large and more complex models [Walker, 1982]. Current trends in management and policy modeling seem to be directed toward the interactive use of relatively smaller interrelated models designed to be adaptive and responsive to a wide variety of questions that policy makers and those affected by a policy may want to ask. Even if each of the smaller models can only address a few questions, together they can be used to study the more complex structured aspects of a resource management problem. The resulting information together with policy makers' experience and judgment about the less structured aspects of the problem can assist in the process of making more informed decisions. This has to be done within the time and budget available and without the need to identify and argue over explicit management or policy objectives [Quade and Miser, 1983; McClure, 1981; House and McLeod, 1977; Hadden, 1982].

Collections of interactively linked models for impact exploration, synthesis, and evaluation are often termed decisionsupport or decision-aiding systems or model management systems [Sprague and Carlson, 1982]. Figure 2 illustrates how a model management or decision-support system approach differs from the approach illustrated in Figure 1. The focus is broader and includes not only models and policy analysts, but reflects also the interactive policy making process. Admittedly, Figure 2 is oversimplified, as is Figure 1, but their comparison demonstrates the change in modeling philosophy now becoming evident.

Figure 2 emphasizes the interaction of managers, planners, and policy makers (or their staffs) with a variety of impactprediction models linked together to their appropriate data bases. An essential feature is the human interaction through a "command" program interface. This gives managers, planners, and policy makers or their staffs the ability to easily explore, define, and synthesize a variety of management or policy options at any time, including during the time when the negotiations over the relative merits of these options are taking place.

This new modeling philosophy recognizes that policy making typically involves a number of actors and interests, conflicting perceptions of nature, contradictory rationalities, and divergent advocacies. Policy making and planning are not a static phenomena, but dynamic historical processes. Complexity, goal ambiguity, contradictory uncertainties, conflict, risk, institutional inertia, and temporal change are not rare characteristics of policy making or planning environments; they are their essential features.

High-level managers, policy makers, and senior bureaucrats are usually where they are because of strongly felt, or at least effectively articulated, beliefs about certain issues. Existing policies, based on those beliefs, are identified with those indi- 


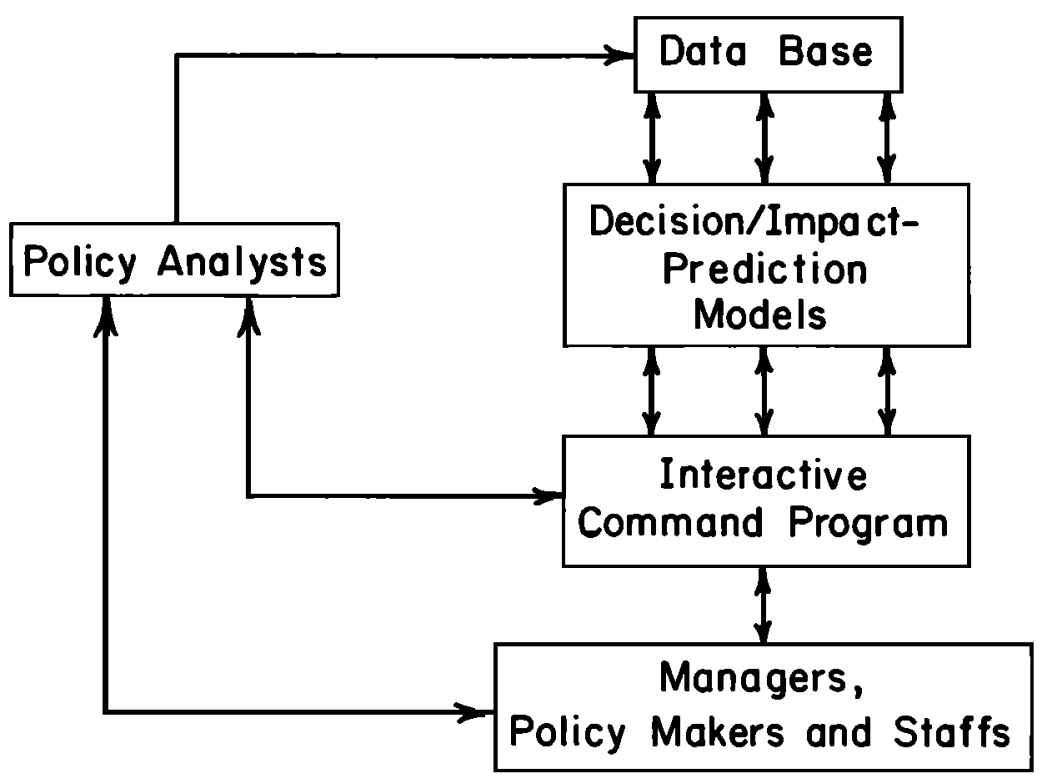

Fig. 2. Interactive relationships among policy makers, policy analysts, their models, and their data, showing strong link between model users, model builders, and those benefiting from the use of models.

viduals. Analysts giving information or providing tools yielding results that question those beliefs and existing policies are not likely to have an audience. The problem is to resist the urge to ignore these features of political life and instead to adapt our thinking and modeling to this fact. Political considerations may permit, at best, incremental or transitional policies that not only preserve reputations but also provide feedback prior to more significant management or policy shifts. We believe that only when modelers include these considerations in their models will their contributions be considered relevant by those individuals and institutions involved in the process of managing, planning, or policy making.

Any shift in an approach to modeling complex resource management problems brings with it new research opportunities. Certainly this is true for the development of systems of interlinked impact prediction models implemented on readily accessible computer work stations [Fedra and Loucks, this issue] possibly coupled to graphical and pictorial input and display devices [Loucks et al., this issue]. Some of the more challenging of these research needs are as follows.

1. Can procedures be developed for deriving systems of simplified, yet sufficiently realistic and reliable predictive models for use in the planning or policy making process? What is the appropriate link between these simplified models and the more comprehensive models that are designed to enhance our scientific understanding of various natural, technological, or social systems? How can systems of relatively simple predictive models representing a complex system be designed to be solved relatively quickly on a small computer and remain credible?

One way of looking at this problem is to realize that all planning or policy models, and especially the more sophisticated ones, are largely determined by their underlying assumptions: what to include and what to leave out, how to describe a given process, which parameter value to choose, how to interpret and prepare empirical data for inputs, etc. Models are thus at least partly based on subjective foundations. In other words, models rely heavily on expert knowledge. It is their heuristic value rather than any degree of precision that makes them helpful in planning and policy making.
The appropriate procedure thus is also a heuristic one: starting from expert knowledge and problem perception, guided by strict parsimony and whatever theoretical framework may be applicable, models are built to satisfy their users' needs for information and enhanced understanding on the planning or policy levels. They should not be built, necessarily, to reliably predict, because how could that be established other than in hindsight?

Model credibility is also a very subjective notion. A model is or is not credible only in the eye of the beholder or modeler. Planning or policy modeling is as much an attempt to depict reality as it is an attempt to formalize and guide our subjective perception and understanding of reality. The only remaining criterion (given a certain professional and scientific standard) is user satisfaction.

Each of the remaining five papers in this series addresses this issue further. The conclusion to be drawn from this admittedly limited sample of experiences and opinions is that the answer to the above question is yes. However, it remains to be seen how this can best be done.

2. How can a system of interlinked models be developed and used so that it contributes not only to increasing the efficiency of any proposed plan or policy or decision (i.e., doing things right) but also to the effectiveness of the policy making process (i.e., helping managers, planners, and policy makers decide what are the appropriate questions to ask and the right things to do)? Related to this is the need to evaluate a wide variety of impacts associated with a large number of objectives or measures of system performance, especially when some of those types of impacts and objectives are not known or thought of until well along in the planning or policy making process.

At the heart of this question is the problem of complexity, of everything affecting everything else. It involves the problem of not knowing what one wants to do, or knowing what will be considered important, until one knows what can be done, or what impacts will result and how they will affect others. In our view, the appropriate approach is a modular one made from numerous more general building blocks in a problemspecific framework. Also, the precondition for such a mode of 
modular model operation is a sufficiently flexible and easy to use software development environment that makes the cost of modular development very small versus the cost of not exploring one more possibility. In different ways, each of the other five papers in this series explores this issue further.

3. How can one build into a model management or decision-support system a means of updating the input data and even the structure of the models, when appropriate, so that the system is adaptive to changing issues, problems and institutional interests or objectives? How can one make the system adaptive to various levels of user sophistication without the need of a full-time computer programmer? Can systems of predictive models be developed and used to provide guidance for, and yet not constrain, the identification of new policy alternatives? This is especially applicable in an environment where not the analysts but the managers, planners, and policy makers (perhaps through their staffs) are asking analysts for information that may not have been considered during the model building stage.

Again, the answer is flexible, easy to modify or reconfigure models, and a development-oriented software environment. A rich set of compatible and cooperating software tools, which can easily be combined and recombined in an experimental fashion, invites the continuous adaptive development of such model systems. Independent but combinable tools, each of them relatively small and easy to grasp, master, and, if necessary, modify or add to, give the user the necessary flexibility at low cost. If it is easy to develop a special purpose ad hoc solution to a given problem (like a special purpose editor program to update or modify input values), the need for necessarily complicated general purpose systems is reduced.

4. How can one most efliciently link various predictive models together when each of these models may have differing and varying spatial and temporal dimensions? The dimensions may depend on the spatial and temporal response of the system being modeled, on the policy or decision taken at each spatial location and in each time period, and on external or exogenous events. Hence the spatial and temporal resolution appropriate to a particular model or module may vary during any simulation.

If individual elements of a model system have different resolutions in space and time, then they can always be linked together with a set of special purpose interface programs, that, in a purely formal or more heuristic way, transform, aggregate, and disaggregate the flow of information between these respective models. Each such interface can be very small, simple, and inexpensive to produce. However, there will be the need to assess how such simplifications distort the information derived from each of the disaggregated models.

5. How can one most effectively combine, contrast, and present information having both spatial and temporal dimensions in a manner that will improve the comprehension of that information by analysts, managers, planners, policy makers, and the interested public?

Interpreting, comparing, and evaluating complex information is a task for the human brain. However, the work of the brain is enhanced if the information is presented in an understandable manner. This, however, may be quite different for different persons. It is therefore necessary to provide a large array of formats and styles, from the more traditional tables of numbers to linguistic statements and color graphics, so that individual users can select interactively what each likes best. Only if the results of formal models are made compatible with the mental models and cognitive style of its users will they be understood and thus contribute to enhanced understanding.

The problem clearly calls for the development of systems designed for use with, and ultimately by, the users in an interactive fashion. If the user can be involved in the design of the interactive system, it can be designed to respond to his or her needs in a more substantive and comprehensible way, using his or her language, symbols, or models of thought. In this respect the papers by Kunreuther and Miller [this issue] and Fedra [this issue] should be contrasted to those of Cosgriff et al. [this issue] and Loucks et al. [this issue].

These and other research issues are important. Many relate to the acceptability of any quantitative modeling or information display system within an essentially political setting. How these problems are or are not overcome in the actual process of building and using interactive models will undoubtedly differ. The interface that links a system of models to model users and the level or extent of interaction needed will be largely dictated by the institutional structure in which the models must fit and adapt as well as by the particular management problems requiring policy.

Hence there are no single answers to these questions. Yet these are some of the questions addressed in the following series of papers on the development and implementation of interactive modeling. Some of our views are the same, some quite different. Some of our work has been successfully implemented; some has not. We leave it to others to critique our ideas and the approaches we have followed, and to add to the experiences reported in these following papers.

The next paper by Kunreuther and Miller [1984] reports on one of the first attempts to develop and apply interactive modeling to a pervasive water problem: flood management and hazard assessment. They began and completed their work in the mid- to late 1970's, and long before anyone seriously thought that those who would most likely be benefiting from the use of such models would have their own personal computers, and in many cases with graphical display capabilities to make the output even more understandable. One can wonder if the difficulties Kunreuther and his colleagues had in the late 1970's in identifying clients who would be interested in using these very innovative tools (a difficult task especially on dry sunny days) will be diminished in the near future. Advances in computer technology will soon make it possible for all of us to have an even more powerful modeling capability linked to appropriate data bases for assessing flood hazards and options for their reduction.

Computer technology is going to have such a profound impact on how we develop and use models that it seems to warrant a more detailed examination. In the next paper, Fedra and Loucks [this issue] speculate on what computer techology will be like in the future and how we as model builders should use this technology for the improvement of water resources and environmental planning, management, and policy making. Any speculation on what this rapidly advancing technology will be like and how it will impact on what we do is risky, but there can be no doubt that it will provide even greater opportunities and potential for making what we do as modelers more relevant and useful to planners and policy makers. We in research must make this happen.

The fourth paper is illustrative of what is taking place in Boston and a few other metropolitan areas; namely, the implementation of computer models for the prediction and real- 
time monitoring and control of water supply systems. One of the most impressive examples of this is being carried out in Melbourne, Australia.

Cosgriff et al. [this issue] in the Melbourne and Metropoli$\tan$ Board of Works (MMBW) have clearly demonstrated the potential advantages and economic benefits that can result from the implementation of such a system of interactive models and graphics display terminals for both analysis and system operation. In our opinion, it is well worth the trip to Melbourne for all having decision-making responsibilities for managing and planning water and sewerage systems to observe MMBW's integrated computer-modeling operation and telemetry capability. What is especially impressive is how a few modelers and computer programmers were not only able to successfully develop such an interactive computer-based system, but also how they succeeded in implementing it in a very complex setting, both physically and institutionally. It is judged by all within MMBW to be a success. It has already saved MMBW six times its initial cost.

Returning to more research-oriented work, Loucks et al. [this issue] review some of the results that they and others at Cornell University have obtained on integrating a variety of water resource and environmental planning and policy models to interactive computer graphics. Of particular interest is the use of interactive computer graphics for assisting in the input, editing, management, and display of spatial and time-varying data. Some of the details associated with the use of reasonably high-resolution color graphics and video-digitizing techniques are discussed and illustrated. This developing technology is becoming increasingly available and less expensive, seemingly with each new issue of any of the numerous journals that describe and advertise this technology. It will be interesting to observe how quickly our profession can learn how best to use these and other similar research results for the improvement of planning and policy making.

The final paper by Fedra [this issue] describes some research on the development of an interactive policy-oriented simulation and information management and display system for a lake region near Vienna, Austria. Fedra's modeling philosophy is somewhat similar to that of Kunreuther and Miller [this issue], which can be contrasted to that reported by Cosgriff et al. [this issue] and by Loucks et al. [this issue], especially with respect to the involvement of the user in the modeling and programming efforts.

\section{CoNCLUSIONS}

The interactive approach to modeling and model use for the exploration, study, and synthesis of resource managment plans or policies is motivated by both a need and by a technology. There is clearly a continuing need for a better means of generating and effectively communicating technical information to those involved in planning and policy making. If models are to be used to do this, we believe such models must be easy to use, easy to understand, and must be adaptive to a wide variety of possible policy alternatives. They must also be able to provide credible information when such information is needed. Furthermore, they should be designed so as to provide an unbiased guide that helps policy makers decide what it is they should do as well as how best to do it.

In addition, the increasing availability and use of timesharing computer networks, microcomputers, and interactive computer languages (software) make it possible for most of us, as individuals or as members of various organizations, to have access to fast, inexpensive, interactive computing power, supplemented increasingly by color display capabilities if desired. These developments will have a profound effect on how we as modelers approach management and policy model building in the future, and how we can help managers, planners, and policy makers better understand technical issues, identify various options that are available for reducing conflict, and to assess the impacts of those alternative policies.

In this first of a series of articles on interactive modeling for water resources and environmental planning and policy making we argue why we think such an approach might lead to more effective modeling and model use. We also focus on some of the problems and research topics related to the development and use or implementation of interactive models. Rather than providing a literature review of the subject [Pollack, 1976], we have attempted to provide some motivation and background for some more detailed discussions of several aspects, and several applications, of interactive systems of models designed to assist and be used by water resource and environmental managers, planners, and policy makers. These more detailed discussions are contained in the following five articles. By no means does the information contained in this and the following papers represent a review of the current state of the art. We are only hoping to add a little to it and to argue for more research and discussion on this topic.

\section{REFERENCES}

Brown, G. E., Jr., Can systems analysis and operations research help Congress?, Interfaces, 12(6), 119-125, 1982.

Cohon, J. L., Multiobjective Programming and Planning, Academic, New York, 1978.

Cosgriff, G. O., P. E. Forte, and M. A. Kennedy, Interactive computer modeling, monitoring, and control of Melbourne's water supply system, Water Resour. Res., this issue.

Fedra, K., A modular interactive simulation system for eutrophication and regional development, Water Resour. Res., this issue.

Fedra, K., and D. P. Loucks, Interactive computer technology for planning and policy modeling, Water Resour. Res., this issue.

Friedman, R. M., C. Ansell, S. Diamond, and N. Ikeda, Use of models for water resources management, planning and policy, Ollice of Technology Assessment, U.S. Congress, Washington, D. C., August 1982.

Goicoechea, A., D. R. Hansen, and L. Duckstein, Multiobjective Decision Analysis With Engineering and Business Applications, John Wiley, New York, 1982.

Hadden, S. G., Scientific data and public policy, Science, 216, 508$509,1982$.

House, P. W., and J. McLeod, Large Scale Models for Policy Evaluation, John Wiley, New York, 1977.

Kahn, H., On Thermonuclear War, Princeton University Press, New Jersey, 1960.

Kunreuther, H., and L. Miller, Interactive computer modeling for policy analysis: The flood hazard problem, Water Resour. Res., this issue.

Lara, F., and W. M. Sachs, Modeling of the environment through a system of models, Appl. Math. Model., 2, 216-219, 1978.

Little, J. D. C., Models and managers: The concept of a decision calculus, Manage. Sci., 16(8), 466-485, 1970.

Loucks, D. P., et al., Research in water resources and environmental systems modeling, Nat. Res. Forum, 8, 219-240, 1984.

Loucks, D. P., M. R. Taylor, and P. N. French, Interactive data management for resource planning and analysis, Water Resour. Res., this issue.

McClure, R. H., Educating the future users of O.R., Interfaces, $I I(5)$, 108-112, 1981.

Pollack, M., Interactive models in operations research-An introduction and some future research directions, Comput. Oper. Res., 1, 305-312, 1976.

Quade, E. S, and H. J. Miser, Handbook of Systems Analyses, International Institute for Applied Systems Analysis, Laxenburg, Austria, 1983. 
Sebenius, J. K., The computer as a mediator, J. Policy Anal. Manage., l(1), 77-95, 1981.

Sprague, R. H., Jr., and E. D. Carlson, Building Effective Decision Support Systems, Englewood Cliffs, Prentice-Hall, New Jersey, 1982. Straus, D. B., and P. B. Clark, Computer-assisted negotiations: Bigger problems need better tools, Environ. Prof., 2, 75-87, 1980.

Walker, W. E., Models in the policy process: Past, present, and future, Interfaces, 12(5), 91-100, 1982.

Weger, C., Graphics help policy makers utilize data, Comput. Graphics News, 2(3), 1-6, 1981.

Whitted, T., Some recent advances in computer graphics, Science, 215 , 767-774, 1982.
Zeleny, M., Notes, ideas and techniques: New vistas of management science, Comput. Oper. Res., 2, 121-125, 1981a.

Zeleny, M., Multiple Criteria Decision Making, McGraw-Hill, New York, $1981 b$.

D. P. Loucks, Hollister Hall, Cornell University, Ithaca, NY 14853.

(Received March 17, 1984;

accepted March 21, 1984.) 

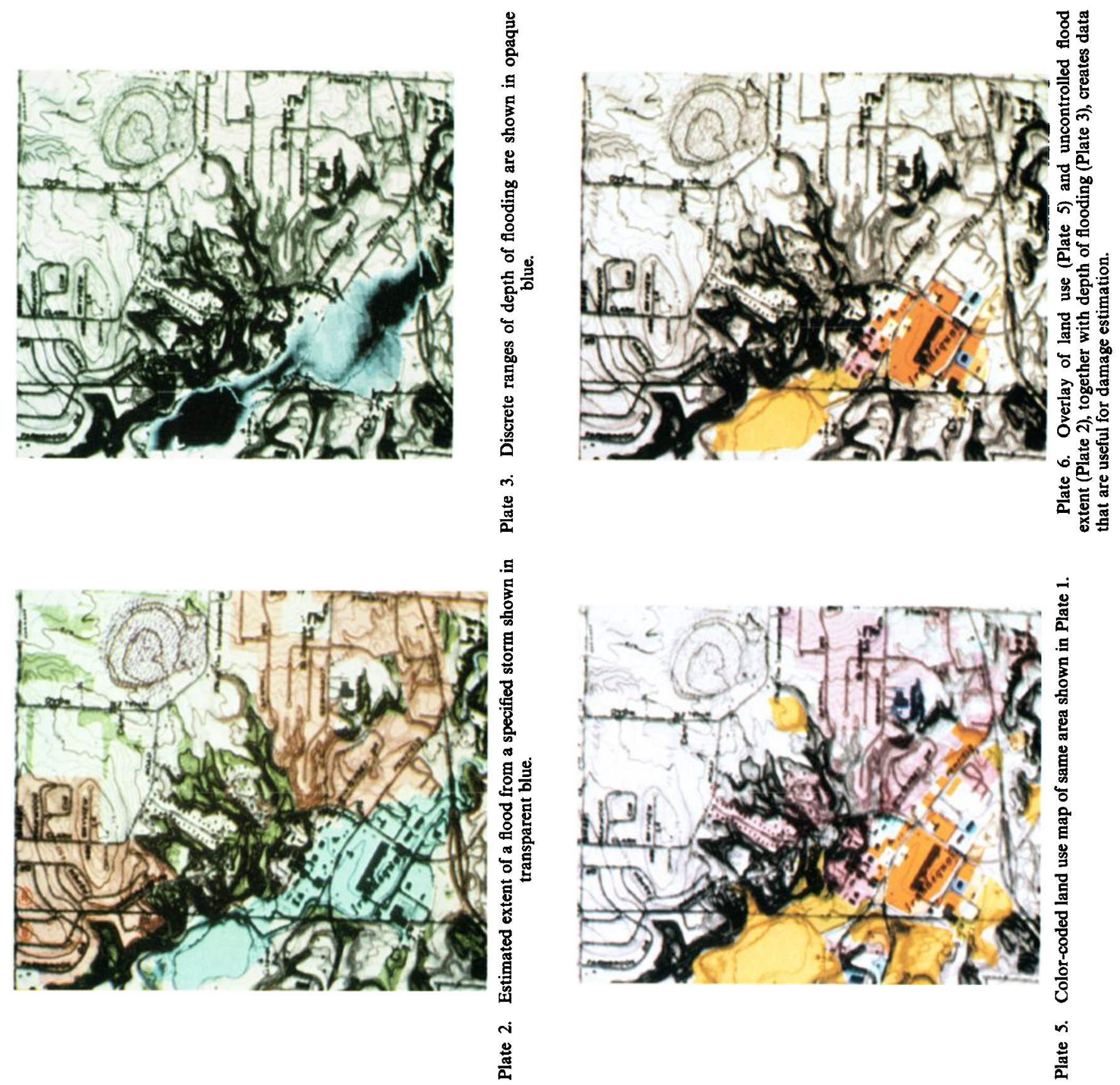

疍曷
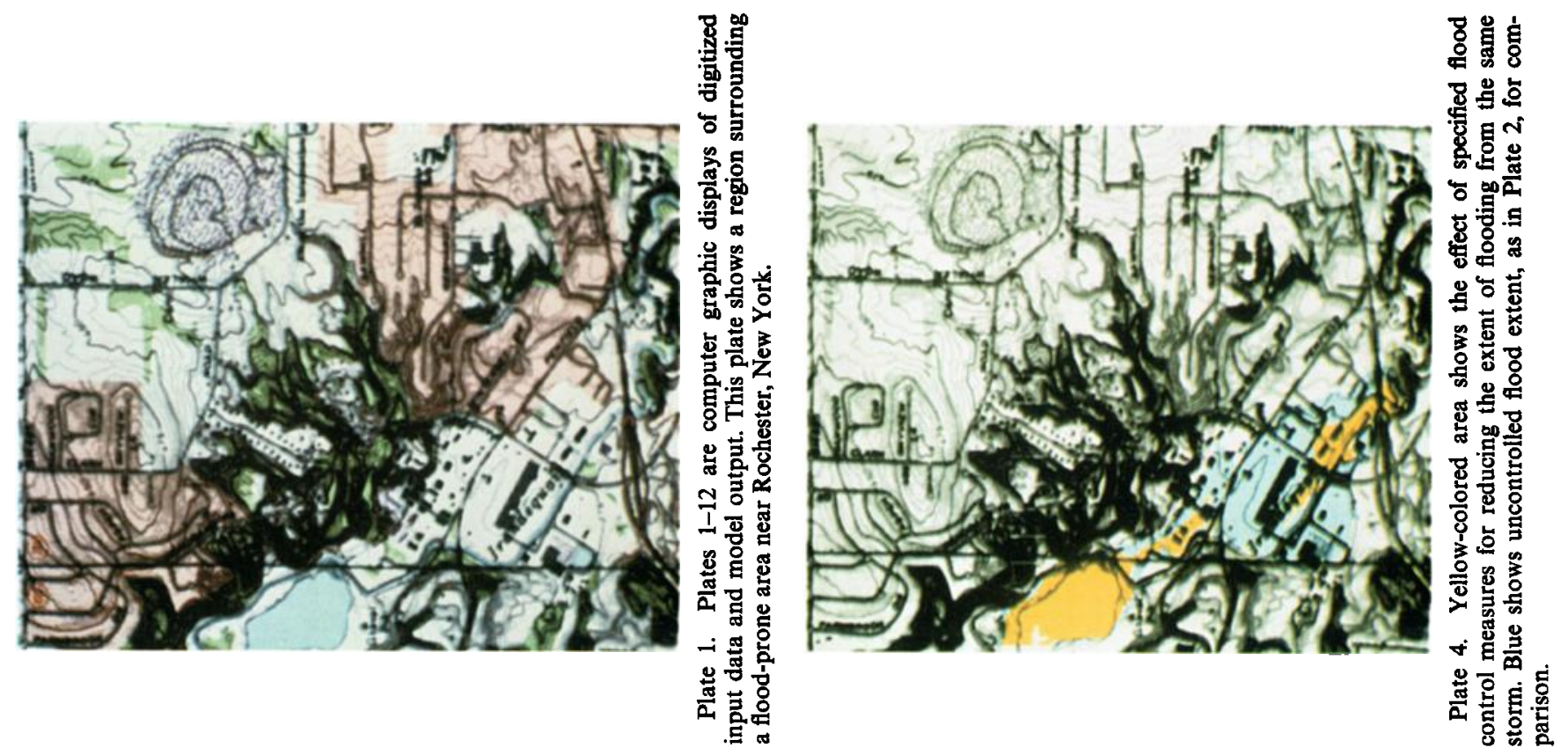


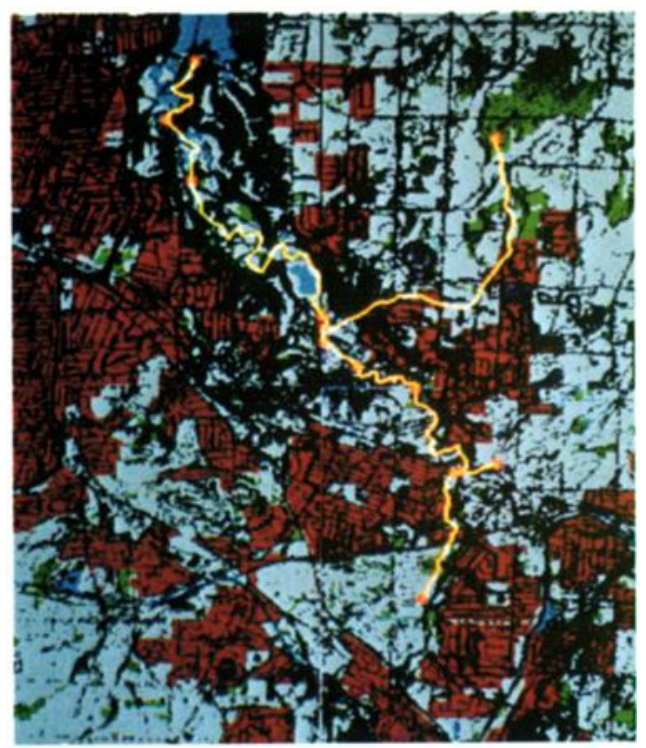

Plate 7. Color-coded predicted stream quality displayed over stream on a darkened digitized map of river basin.
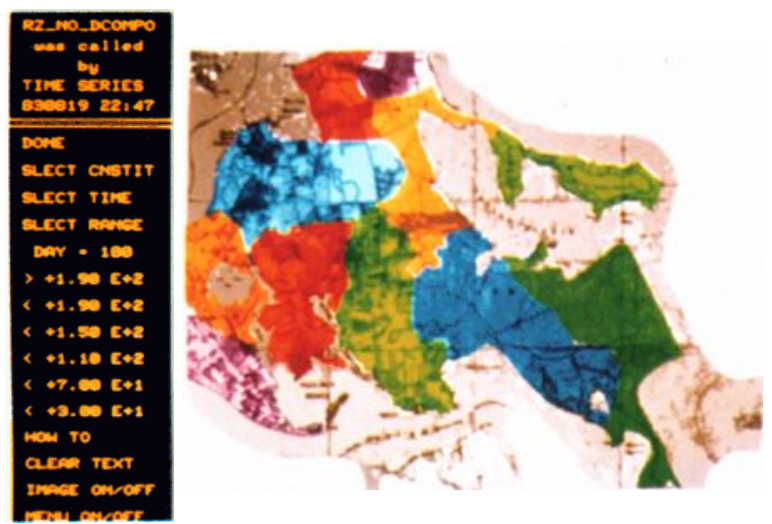

Plate 9. Ranges of nitrogen concentrations in the unsaturated subsurface zones of a portion of Long Island are shown in different colors, as defined in menu section of the display.

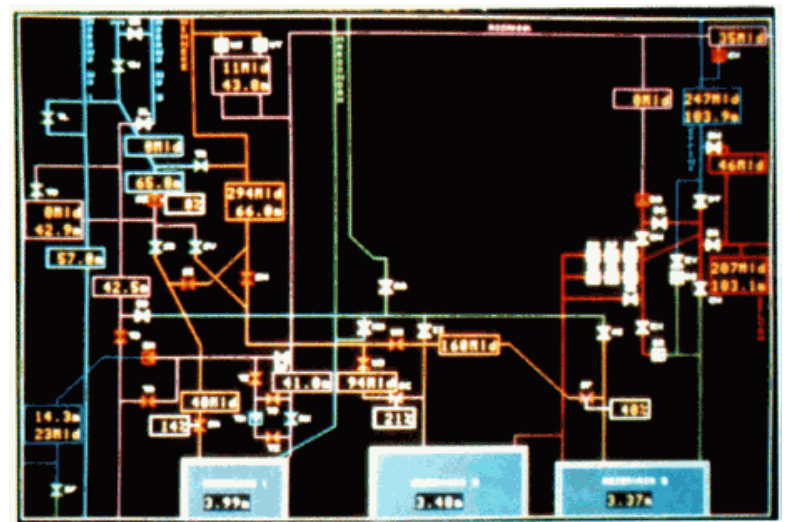

Plate 11. A schematic of a portion of Melbourne's water supply system showing actual status of flows, valve settings, and reservoir volumes.

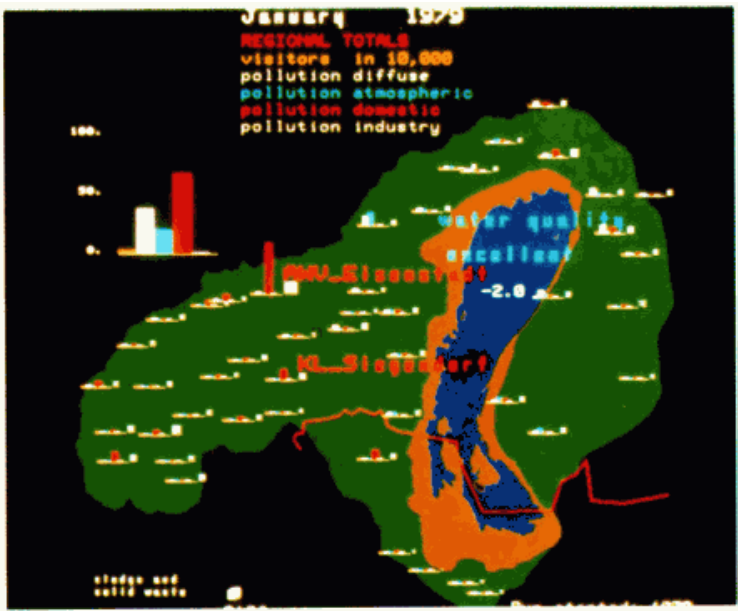

Plate 8. Lake Neusiedl watershed on the Austrian-Hungarian border (red line) showing data from simulation of interaction between tourist and agriculture development and lake water quality.

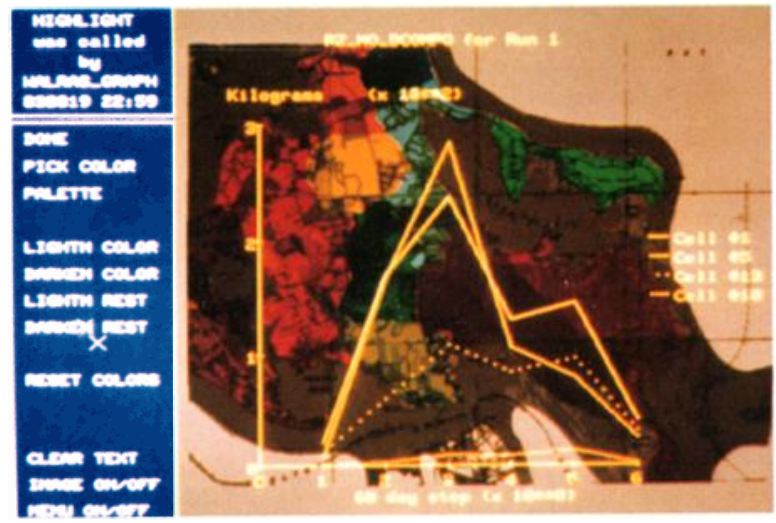

Plate 10. Graphical and alphanumeric information can be displayed over a darkened raster image for ease in interactively analyzing various spatial and time varying phenomena.

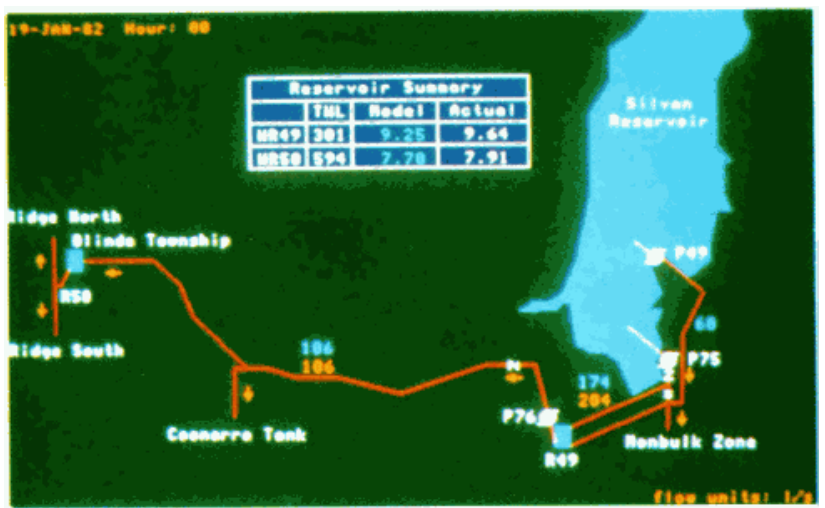

Plate 12. A schematic of a portıon of Melbourne's water transter system from a major reservoir to the distribution system showing modeled and actual operational data. 